drift at 3-month follow-up was $1.2 \pm 1.3 \mathrm{mmHg}$ per month in seven patients; greater drift as a result of a device fault in one patient was corrected by noninvasive calibration. No deaths or complications adjudicated to be device-related occurred during the 3-month follow-up, and none of the patients made an unplanned visit to the hospital for symptoms relating to decompensated HF during this period. In total, patients recorded $92 \pm 8 \%$ of the scheduled twice-daily measurements.

The researchers conclude that the new implantable LAP monitoring device is safe and can accurately measure LAP in ambulatory patients with HF. Further studies of this device are warranted to determine whether ambulatory LAP monitoring can improve outcomes in HF.

Original article Ritzema J et al. (2007) Direct left atrial pressure monitoring in ambulatory heart failure patients: initial experience with a new permanent implantable device. Circulation 116: 2952-2959

\section{Catheter ablation reduces the incidence of shocks from ICDs}

Implantable cardioverter-defibrillators (ICDs) are the mainstay of treatment for patients at risk of ventricular tachycardia or fibrillation. Although these devices reduce mortality, defibrillator discharges are painful, and several studies have reported an association between recurrent ICD shocks and anxiety and depression. Now, Reddy et al. have shown that prophylactic substrate-based catheter ablation can reduce the incidence of ICD shock and antitachycardia pacing in patients who have received an ICD for a ventricular tachyarrhythmic event.

In this prospective, unblinded, multicenter trial, 128 patients with an ICD were randomized to an ablation or control (no ablation) group on a 1:1 basis. There were no deaths in the ablation group at 30 days, although substantial ablation-related complications occurred in three patients. No patients received antiarrhythmic medication during the trial (other than $\beta$-blockers) before they had experienced an ICD event.

After 2 years of follow-up, the proportion of patients who had received at least one ICD shock or episode of antitachycardia pacing was significantly lower in the ablation group (12\%) than in the control group $(33 \%$; hazard ratio
0.35, 95\% Cl 0.15-0.78), and, among these individuals, fewer patients in the ablation group received ICD shocks ( $9 \%$ vs $31 \% ; P=0.003$ ). Furthermore, the probability of receiving ICD therapy was lower in the ablation group than in the control group even following adjustment for patient characteristics and medication use at baseline (hazard ratio $0.31,95 \% \mathrm{Cl} 0.13-0.76$ ). Notably, ablation was not associated with any appreciable change in ventricular function or NYHA functional class during follow-up, even in those patients with the most depressed cardiac function.

Original article Reddy VY et al. (2007) Prophylactic catheter ablation for the prevention of defibrillator therapy. N Engl JMed 357: 2657-2665

\section{Study highlights poor adherence to cardiovascular treatment goals in primary care}

Published guidelines for the management of cardiovascular disease recommend treatment goals for the key modifiable risk factors. Whether such goals are successfully attained in primary care is, however, largely unknown. Geller et al. assessed this feature in Germany in a large patient cohort.

The researchers used the patient management systems of 110 physician offices in Germany to extract data on all patients $(n=715,844)$ seen from January 1998 to June 2005. The study population for this investigation comprised the 284,096 (39.7\%) individuals who had cardiovascular disease or diabetes. Among this population, $55.3 \%$ had hypertension, $22.6 \%$ had coronary artery disease, $29.2 \%$ had diabetes, $61.5 \%$ had hyperlipidemia and $48 \%$ had more than one of these conditions. At the last clinic visit, treatment goals for total and LDL cholesterol were reached in only $9 \%$ and $29 \%$ of patients, respectively. Furthermore, only $28 \%$ of the patients who had been diagnosed with hypertension had blood pressure values within the desired range, and only $36 \%$ of the patients diagnosed with diabetes had glycated hemoglobin levels below the recommended threshold. Similarly poor adherence to treatment goals was seen in high-risk patients (defined as those with coronary artery disease and/or diabetes), with $<10 \%$ and $14 \%$ of this subgroup achieving treatment goals for total and 